\title{
On-site treatment of exertional heat stroke
}

Brian K. Sloan, MD, Emily M. Kraft, MD, Dave Clark, Scott W. Schmeissing, MD, Brian C. Byrne, MD and Daniel E. Rusyniak, MD

\section{Background}

ABSTRACT

Exertional heat stroke is a devastating condition that can cause significant morbidity and mortality. Rapid cooling is the most effective means of treating heat stroke but little is published on the safety and logistics of cooling patients on-site at a major sporting event.

\section{Purpose}

To describe an on-site exertional heat stroke (EHS) treatment protocol and to compare the outcomes of patients treated on-site to those transferred to hospitals.

\section{Study Design}

Descriptive Epidemiological Study

\section{Methods}

Using race day medical records and ambulance run sheets, we identified patients who developed exertional heat stroke at the Indianapolis half marathon from the years of 2005-2012. EHS was defined as runners with a core temperature measured with a rectal thermometer greater than $102^{\circ} \mathrm{F}$ and altered mental status. Clinical information and patient outcomes were abstracted from the race medical tent and hospital charts by three separate trained reviewers using structured methods and a data collection form. Three reviewers, using a RedCAP database and dual-data entry, abstracted records for each patient. A third reviewer arbitrated all discrepancies between reviewers. Clinical signs, treatments, and outcomes were calculated using descriptive statistics and data were grouped and compared for patients treated on-site or transferred to local hospitals for treatment.

\section{Results}

Over 235,000 athletes participated in the event over the eight-year period with 696 seeking medical care. Thirty-two heat stroke victims were identified during the study period and of these 22 were treated on-site. Of these $68 \%$ were treated with cold-water immersion and $59 \%$ of were discharged home from the race. Ten exertional heat stroke patients were transported from the

This is the author's manuscript of the article published in final edited form as:

Sloan, B. K., Kraft, E. M., Clark, D., Schmeissing, S. W., Byrne, B. C., \& Rusyniak, D. E. (2015). On-site treatment of exertional heat stroke. The American Journal of Sports Medicine, 43(4), 823-829. 
racecourse to local hospitals. None of them underwent cold-water immersion and $40 \%$ of them were subsequently discharged home. No patients in the study died.

\section{Conclusions}

On-site treatment of athletes that develop EHS appears to be both safe and effective. On-site treatment may decrease the local burden of critically ill patients to emergency departments during large athletic events.

\section{Key Terms}

Exertional heatstroke, cold water immersion, cooling, road race

What is known about this topic?

Rapid cooling of EHS patients is the most effective means to prevent morbidity and mortality. Of available methods, cold-water immersion has been shown to be the most effective.

\section{What this study adds to existing knowledge?}

We show that on-site cooling of EHS patients is logistically feasible and effective. Rapid on-site cooling of EHS patients can decrease hospital burden on race day and minimize participant hospitalizations and costs. 


\section{Introduction}

Over the last 20 years, road races such as triathlons, marathons, and half marathons have increased in popularity. In 2013 alone, there were 19 million participants involved in 28,200 road races. ${ }^{20}$ Many of these events take place in conditions that predispose individuals to developing exertional heat stroke (EHS). EHS is the second most common cause of non-traumatic death in competitive athletes. ${ }^{15}$ In fact, for every serious cardiac adverse event at races there are 10 serious cases of heat stroke. ${ }^{22}$ The most effective treatment for EHS is rapidly cooling the patient. ${ }^{18}$ Delays in cooling can lead to morbidity and mortality. ${ }^{4}$

A variety of cooling methods have been used in the treatment of EHS: ice massage; cooling blankets; cold towels; cool air exposure; ice packs to axilla, groin, and neck; water spray in a body cooling unit; and cold water immersion. ${ }^{2,12,13,16}$ Of these, the most rapid means of lowering core temperature is cold-water immersion (CWI). ${ }^{7,}$ In the hospital emergency department (ED) cold-water immersion may not be practical. CWI requires immersion tubs, adequate space, and educated nurses, physicians, and ancillary staff. Even in EDs that have immersion tubs, a road race on a hot day can result in multiple heat stroke victims that can quickly overwhelm hospital resources. Additionally, since the outcome of heat stroke patients is dependent on the speed at which their core temperature can be lowered, ${ }^{18}$ the time it takes to transport a patient to a local ED is time lost that could be spent cooling them.

On-site treatment of EHS at major sporting events offers the safest and most efficacious means to rapidly cool patients. Resources can be prepared ahead of time, staff can be trained, and facilities can be designed to accommodate a large number of patients. Numerous organizations including the National Athletic Trainers Association (NATA), ${ }^{5}$ the American College of Sports Medicine (ACSM), ${ }^{1}$ the American Heart Association and American Red Cross, ${ }^{14}$ and the 
American Academy of Pediatrics (AAP), ${ }^{3}$ encourage on-site treatment of exertional heat illness in athletes. In spite of these, no previous papers have described the effectiveness, safety, and logistics of treating exertional heat stroke on-site.

This paper describes our experience at the Indianapolis Mini-Marathon, the largest half marathon in the United States. In 2005 we developed a protocol for the on-site event treatment of exertional heat stroke. Our protocol incorporated an easy and cost-effective treatment plan that incorporates CWI. In this descriptive epidemiologic study we review our protocol and describe the presentations and outcomes of patients treated on-site for exertional heat stroke. By means of comparison we also describe treatment of patients off-site (via direct hospital transport).

\section{Materials and Methods}

\section{Medical Coverage at the Indianapolis Mini-Marathon}

The Indianapolis Mini-Marathon is a 13.1-mile running race held the first weekend of May in Indianapolis, IN. With over 30,000 participants each year, it is the largest half-marathon in the United States. The course consists of a 13.1 mile running race through the streets of the city with the middle portion (miles 6-8) of the course run around a 2.5-mile Motor Speedway oval. Two venues provide medical care at the race. One is located at the motor speedway hospital (IMS). It is an 18-bed hospital staffed by eight nurses and two physicians. Patients who are in need of medical care while running on the IMS track can self-triage to the hospital or if they develop emergent conditions may be brought to the track hospital by EMS stationed around the oval. The other location for medical treatment is located at the race course finish line and consists of four medical tents: triage, immediate care, minimal care, and cooling (Fig 1). It is staffed with 18 nurses, 4 paramedics, 8 physical therapists, one podiatrist, and four emergency physicians. Patients who develop medical problems at or after the finish line can self-triage or if they 
develop emergent conditions between Mile 12 and the finish can be brought in via EMS. Outside of the two medical venues, emergency medical providers (EMS) are located at each mile of the race to respond to emergent conditions (e.g., cardiac arrest, syncope, orthopedic injury). Runners suffering from emergent conditions outside of the IMS or before the last mile of the race are transported by EMS to one of two referral hospitals. Both are Level-1 trauma centers with $>100,000$ patient visits a year. The hospitals are located similar distances from the race course.

\section{Exertional Heat Stroke Protocol}

In 2005, the team providing medical coverage for the Indy 500 Mini-Marathon instituted an onsite treatment protocol for participants who develop exertional heat stroke during the last mile of the race or after race completion. A flow diagram of the treatment protocol is represented in Figure 2. Any patient brought by EMS to the medical triage tent who had a palpable pulse and an altered mental status was immediately transported to the cooling tent (Fig 1). On arrival at the cooling tent, patients had their core temperature taken, an intravenous line placed, and blood drawn for point of care testing of sodium and glucose (i-STAT®, Abbott Diagnostics). Any patient who had a temperature $>102^{\circ} \mathrm{F}$ and altered mental status was taken to a cooling tub (Fig 3). Cooling tubs consisted of Rubbermaid ${ }^{\circledR} 50$ gallon horse troughs filled with room temperature water and ice. Prior to submersion, the majority of patients had a rectal thermometer inserted, and an intravenous infusion with room temperature normal saline was begun. Patients were removed from the tub when their core temperatures were approximately $102^{\circ} \mathrm{F}$ or when their mental status returned to normal. In the event that the number of patients exceeded either the number of available cooling tubs or the number of staff that could man them, patients were cooled with ice packs to the axilla, groin, and neck and intravenous fluids. Independent of 
treatment, those patients who reached the target cooling temperature and still had altered mental status or abnormal vital signs were transported via EMS to the hospital. Patients who reached the target cooling temperature and had normal mental status and vital signs were discharged home. This cooling protocol was not instituted at the IMS hospital. Any patient presenting to this medical facility was treated at the discretion of the IMS hospital personnel.

\section{Design and Data Abstraction}

We conducted a retrospective epidemiologic study of all patients who developed exertional heat stroke at the Indianapolis Mini-Marathon. This included both patients treated on-site at the medical tents, using our previously described exertional heat stroke protocol, and those treated off-site (those treated at the IMS hospital or treated at one of the two local hospitals). The Institutional review board of Indiana University School of Medicine approved this study.

Patients were identified using medical charts and EMS run sheets. Both the medical tents and the IMS hospital used medical charts to record patient information, clinician findings, treatment, and disposition. In addition, all EMS transports from the medical tents, the IMS hospital, or from the race course were logged and include the ambulance run number, the patient name, and the location to where the patient was transported. An investigator reviewed all the medical records from patients presenting to the medical tents or the IMS hospital between the years of 2005 and 2012. In addition, investigators reviewed records of any patient transported from the race to a local hospital. Patient records with a recorded body temperature of $>102^{\circ} \mathrm{F}$ or altered mental status were binned for further data analysis.

Study data were collected and managed using REDCap electronic data capture tools hosted at Indiana University School of Medicine. ${ }^{11}$ As described on their website "REDCap 
(Research Electronic Data Capture) is a secure, web-based application designed to support data capture for research studies, providing 1) an intuitive interface for validated data entry; 2) audit trails for tracking data manipulation and export procedures; 3) automated export procedures for seamless data downloads to common statistical packages; and 4) procedures for importing data from external sources”. Data collection forms were created for both on-site treatment at the medical tents and off-site treatment at the IMS or local hospitals. Both data collection forms are included in Appendix A. Briefly, data consisted of basic demographic information, vital signs on arrival and after treatment, blood sodium and glucose, cooling modality, and disposition. All the data was stored on a password-protected server and identifying patient information was removed before final analysis.

Three data abstractors independently reviewed the binned records from the medical tent and IMS hospital and, for those patients transported, individual hospital records. Each patient had data abstracted by two reviewers. Using the dual-data entry feature in REDCap, records were compared from each abstractor and discrepancies arbitrated by a third party review of the chart. The final compiled records were then reviewed and patients who met our a priori definition of exertional heat stroke that underwent treatment in the medical tent area using our heat stroke protocol were analyzed using descriptive statistics (Microsoft Excel, Microsoft Seattle, WA). For data analysis we defined exertional heat stroke as any patient with a recorded temperature of $\geq$ $102^{\circ} \mathrm{F}$ and altered mental status. While there are variable definitions for exertional heat stroke we purposely chose a more inclusive definition to account for confounders that are inherent to retrospective analysis. For instance, while all medical providers in the tent area were instructed to obtain rectal temperatures, this did not always occur. Oral and axillary temperatures were recorded, or the mode of temperature measurement was not always reported. Therefore to insure 
we did not miss any patients, we purposely chose a temperature lower than most authors report. We choose to broadly define altered mental status as patients who had any of the following recorded in their medical record: delirium, confusion, somnolence, coma, seizure, altered mental status, a recorded Glasgow Coma Scale $\leq 14$, stuporous, lethargic, obtunded, unconscious, syncope, passed out, combative, uncooperative, collapsed, or unresponsive.

To assure that all patients with exertional heat stroke were captured we cross-referenced the medical tent records and IMS hospital records with the entire EMS run sheets to identify every patient transferred to the hospital on half marathon race day. We reviewed the medical records for each of these patients to make sure no heat stroke patients treated at the medical tent were missed. A search of hospital records did not identify heat-illness patients who had not been seen by on-site personnel.

\section{Results}

Race Demographics

Between 2005 and 2012 over 235,000 persons participated in the race. Of these, 696 patients sought on-site medical attention (Table 1) and 22 of these met our definition of exertional heat stroke. In addition, 10 patients transported to local hospitals had exertional heat stroke.

Collectively this yields an event rate of 1.3/10,000 participants. The majority of exertional heat stroke cases (78\%) occurred on the three race days with the highest ambient temperature (Table 1).

Heat stroke cases managed on-site 
Of the 32 patients who developed heat stroke during our study period, 22 (69\%) were managed on-site at the race finish line medical tents (Table 2). The patients core temperatures ranged from $102.4^{\circ} \mathrm{F}$ to $108.5^{\circ} \mathrm{F}$. Thirty five percent of patients had a documented rectal temperature and in $52 \%$ of the cases the mode of temperature measurement was not documented. The majority of patients (85\%) were tachycardic (HR>100 bpm). Only 41\% had hypotension (systolic blood pressure lower than $100 \mathrm{~mm} / \mathrm{hg}$ ) and no patient had blood pressure $<70 \mathrm{~mm} / \mathrm{hg}$. The majority of patients were treated with cold-water immersion (68\%) with the remaining patients treated with ice packs (neck, axilla, groin), cool IV fluids, cold towels, or a combination of these modalities. The majority of patients treated on-site for heat stroke (59\%) were released from the medical tent to home. Of the nine remaining patients, six were seen in a local ED and released home, two were admitted for overnight observation, and one was admitted to the ICU. All patients transferred from the medical tent and admitted to the hospital had good outcomes. Compared to an on-site admission rate of $41 \%$, patients treated off site had an admission rate of $60 \%$. No patients died from heat stroke.

\section{Heat stroke cases managed off-site}

We identified ten patients that suffered exertional heat stroke who because of proximity to the Indianapolis Motor Speedway hospital (Mile 7 of the race) or because of distance to the medical tents (all cases occurring prior to Mile 12) were treated outside of the medical tents (Table 3): One patient was treated at the track hospital and 9 patients were transported by EMS directly to a local hospital. Similar to patients treated in the medical tents these patients had a wide range of temperatures $102.8^{\circ} \mathrm{F}$ to $107.9^{\circ} \mathrm{F}$. All of the patients were tachycardic (HR $\left.>100 \mathrm{bpm}\right)$ and two patients had a SBP $<100 \mathrm{mmHg}$. No patient treated off-site was cooled using cold-water 
immersion. Instead ice, cool mist, cool IVF, fans and in one patient a medical cooling device was used (Arctic Sun, Louisville, CO) to lower body temperature. Patients treated off-site had a hospital admission rate of $60 \%$, with two patients admitted to the ICU; one of these patients was intubated and mechanically ventilated in the ICU for 6 days. No patient treated died and all had good clinical outcomes.

\section{Discussion}

Position statements from many health organizations encourage on-site treatment of exertional heat stroke in athletes ${ }^{1,3,5,14}$. The use of an on-site-treatment protocol for athletes who developed exertional heat stroke at the Indianapolis Mini-Marathon was safe and effective. No deaths from exertional heat stroke occurred during the study period despite patients having core body temperatures as high as $108.5^{\circ} \mathrm{F}$. Perhaps a more surprising result from our data is that the majority of patients treated on-site for heat stroke were discharged home. It is our general practice that patients can be discharged to home from the medical treatment area when they are asymptomatic, have normal vitals signs, are ambulatory, can take oral fluids, and have safe transportation.

While our study was not designed to directly compare outcomes between patients treated on-site and off-site, there was a trend toward a higher rate of hospital admission in those treated off-site. Although our off-site hospitals were within one mile of the race venue, the time it takes to identify the heat stroke patient, transport them to the hospital, and begin treatment in the ED is time the patient is not being effectively cooled. Furthermore, few hospitals are prepared for CWI. In our study, no patient treated off-site underwent CWI treatment. Despite our preparation, some patients with exertional heat stroke did not undergo CWI in the race medical tents. We were unable to determine why this occurred. It is possible that a surge of heat stroke cases 
overwhelmed our supply tanks or the personnel manning them. Another possibility is that patients were too combative to be placed in a CWI tank. In future studies it will be important to identify and eliminate reasons patients do not undergo CWI. Most of those patients not treated with CWI had ice packs placed over large vessels that, although this is a slower method of cooling than $\mathrm{CWI}^{6}$ it also appeared to be effective.

Previous studies have shown that the faster you can cool someone's body temperature the better their clinical outcome. ${ }^{5} \mathrm{CWI}$ has been shown to be the most rapid means to do this. Coldwater immersion however is a logistical challenge requiring tubs, space, cold-water, and personnel dedicated to overseeing the treatment. Because of this, cooling patients with CWI is most effectively done on-site. We utilized inexpensive 50-gallon Rubbermaid ${ }^{\circledR}$ horse troughs, purchased at a local farm supply store, to cool patients. The tubs are prefilled with room temperature water and ice is added when patients are partially submerged. On-site treatment of heat stroke patients also decreases the number of subjects transported to local emergency departments (ED). Because it is difficult to predict when heat stroke cases will occur local EDs may not be prepared to care for a surge of critically ill heat-related patients. Having a large number of trained and prepared health care providers on-site not only allowed for more rapid identification and treatment of patients but it also decreased the number of patients needing hospital transport. The majority of patients treated on-site for heat stroke were able to go home from the race without further intervention. Although a large event like ours requires a significant number of highly skilled nurses, paramedics, and physicians we have never had difficultyrecruiting volunteers for this event. Because all of the staff are volunteers, treatment administered on-site is also cost effective. 
When we first developed our protocol we had concerns that medical personnel would be uncomfortable delaying the hospital transfer of the critically ill hyperthermic, unresponsive, and hypotensive patient to immerse them in cold water. Anecdotally we have found this not to be the case. Rather, having witnessed a number of patients rapidly regain normal mental status and vital signs, our volunteer medical personnel are comfortable and now strong advocates for on-site treatment. However, we have been challenged with poor documentation with many of the patients that were treated at the half marathon event. One of the areas this was witnessed was with the documentation of the mode of temperature recording during the EHS patients' treatment. Because the medical personnel caring for the patients are often the ones who do the documentation the method of temperature measurement was often not recorded. Anecdotally, the authors, who work at the event, have noted that protocol deviation is rare and rectal temperatures are typically used for clinical decision making. To facilitate better collection we now employ volunteers at each event to perform documentation.

The incidence of heat stroke increases with increases in ambient temperature. ${ }^{7,9}$ However as shown in this paper, and in other reports, ${ }^{19}$ exertional heat stroke can still occur on "relatively" cool days. In our study the highest number of heat stroke cases occurred on a day where the $10 \mathrm{am}$ temperature was $64.9^{\circ} \mathrm{F}\left(18.3^{\circ} \mathrm{C}\right)$. In addition to the weather on the race day the athlete's temperature acclimation is likely a contributing factor. ${ }^{21}$ For instance, those training at a temperature and humidity lower than those on race day may be at increased risk for heat stroke even in temperate conditions. The weather in the Midwest in the beginning of May, the time the Indianapolis Mini-Marathon is held, is highly variable. As it is estimated that it takes two weeks to acclimate to exertion in a warm environment ${ }^{21}$ it may be important to know the difference 
between the training temperature and race temperature in making preparations for potential heat stroke cases. Further studies are needed to confirm this.

There are many additional non-weather related risk factors for developing exertional heat stroke. ${ }^{1}$ Included in this are genetic predisposition, ${ }^{17}$ hydration status, sleep status, and medications. ${ }^{8}$ Future studies or registries should include these factors to further our understanding of risk factors involved in the development of exertional heat stroke.

Our protocol proved to be safe and effective in treating persons with exertional heat stroke. For those interested in adopting a similar protocol we recommend including the following:

1. A triage system that rapidly identifies heat stroke victims.

2. Have CWI equipment available and trained personnel in place.

3. Have backup cooling plans in case CWI protocol is overwhelmed.

4. Develop a strategy with local EMS for transports and discuss with local hospital EDs.

5. Have the capacity to alert participants when conditions are favorable for developing heat related illness.

6. Provide a public service message to runners to alert them on the factors that predispose to heat related illness.

\section{Conclusion}

On-site treatment of exertional heat stroke is safe and effective and may decrease the need to transport patients to the hospital. A similar approach could be adapted for football practice, large mass gathering events in the heat, military training, and for firefighters. Future studies are needed to help predict what race conditions and medical factors further increase the risk for athletes developing heatstroke. 


\section{Figure Legends}

Figure 1 Map of on-site treatment area

The on-site medical treatment area consisted of 4 tents (triage, immediate and delayed care,

minimal, and cooling). The arrows represent the flow of patients to and after triage. The cooling tent consisted of 8 cooling tubs and cots represented by ovals and rectangles respectively.

Figure 2 Flow diagram of on-site treatment protocol for patients with altered mental status at the Indianapolis Mini-Marathon.

Italicized text represents diagnostic testing, bold text represents interventions. The rectangle is the entry into the altered mental status algorithm. Diamonds represent decisions points and ovals dispositions.

Figure 3 Photo of cold-water immersion tub and patient undergoing treatment. 
Table 1. Race Statistics

\begin{tabular}{l|l|l|l|l|l|l}
\hline Year & $\begin{array}{l}\text { Race } \\
\text { Finishers }\end{array}$ & $\begin{array}{l}\text { Medical } \\
\text { Patients }^{\mathbf{a}}\end{array}$ & $\begin{array}{l}\text { Heat } \\
\text { Stroke }^{\mathbf{b}}\end{array}$ & $\begin{array}{l}\text { Race } \\
\text { Temp (F) }^{\mathbf{c}}\end{array}$ & $\begin{array}{l}\text { Race } \\
\text { Humidityc }^{\mathbf{c}}\end{array}$ & $\begin{array}{l}\text { Race } \\
\text { Conditions }^{\mathbf{c}}\end{array}$ \\
\hline 2005 & 25,028 & 127 & 7 & $66.9^{\circ}$ & $57 \%$ & Scattered Clouds \\
2006 & 27,644 & 60 & 0 & $53.1^{\circ}$ & $57 \%$ & Scattered Clouds \\
2007 & 29,213 & 63 & 10 & $64.9^{\circ}$ & $81 \%$ & Mostly Cloudy \\
2008 & 30,063 & 98 & 3 & $64.0^{\circ}$ & $63 \%$ & Scattered Clouds \\
2009 & 30,281 & 60 & 1 & $53.1^{\circ}$ & $74 \%$ & Mostly Cloudy \\
2010 & 31,047 & 36 & 0 & $48.9^{\circ}$ & $59 \%$ & Mostly Cloudy \\
2011 & 30,649 & 95 & 3 & $55.0^{\circ}$ & $80 \%$ & Light Rain \\
2012 & 31,129 & 160 & 7 & $71.1^{\circ}$ & $81 \%$ & Scattered Clouds \\
\hline
\end{tabular}

aAny patient cared for at the medical tent or IMS hospital

${ }^{b}$ Heat stroke defined as temperature $\geq 102^{\circ} \mathrm{F}$ and altered mental status

cTemp, Humidity, and conditions at 10 am on race day obtained from http://www.wunderground.com. Race began between 7-8a. 
Table 2. Patients treated on-site

\begin{tabular}{|c|c|c|c|c|c|}
\hline Year & Temp & HR & SBP & Treatment & Disposition \\
\hline 2005 & 108.5 & 129 & 88 & CWI & Hospital \\
\hline 2007 & 108.4 & unk & unk & CWI & Hospital \\
\hline 2007 & 107.5 & 109 & 122 & CWI & Hospital \\
\hline 2008 & 107.5 & 160 & 120 & CWI & Home \\
\hline 2005 & 107.2 & 140 & 94 & CWI & Home \\
\hline 2007 & 107.1 & 132 & 110 & Ice & Home \\
\hline 2012 & 106.9 & 126 & 114 & CWI & Home \\
\hline 2012 & 106.8 & 113 & 83 & IVF & Home \\
\hline 2007 & 106.4 & 108 & 97 & CWI & Hospital \\
\hline 2007 & 106.4 & 123 & 117 & CWI & Hospital \\
\hline 2007 & 106.3 & 135 & 80 & CWI & Home \\
\hline 2012 & 105.8 & 164 & 102 & CWI & Hospital \\
\hline 2012 & 105.0 & 99 & 156 & Ice, IVF & Home \\
\hline 2012 & 104.8 & 137 & 92 & IVF & Home \\
\hline 2007 & 104.4 & 150 & 98 & CWI & Home \\
\hline 2009 & 104.4 & 144 & 166 & CWI & Hospital \\
\hline 2007 & 103.8 & 112 & 89 & Ice & Hospital \\
\hline 2007 & 103.6 & 92 & 110 & unk & Hospital \\
\hline 2005 & 103.2 & 105 & 86 & CWI & Home \\
\hline 2011 & 103.2 & 134 & 113 & Ice, Towels, IVF & Home \\
\hline 2011 & 103.2 & 129 & 101 & CWI & Home \\
\hline 2011 & 102.4 & 146 & 130 & CWI & Home \\
\hline
\end{tabular}

CWI = Cold Water Immersion; Ice = Ice packs to groin, axilla, neck; IVF = Cool IV fluids; Towels $=$ Cold Towels; unk $=$ unknown 
Table 3. Patients treated off-site

\begin{tabular}{c|l|c|c|l|l|l}
\hline Year & Mile & Temp & HR & SBP & Treatment & Disposition \\
\hline 2012 & unk & 107.9 & 168 & 129 & IVF, Ice, MCD & ICU \\
2008 & 11 & 107.2 & 149 & 100 & Fans & Admit \\
2012 & 7 & $107.0^{\text {a }}$ & 138 & 110 & IVF, Ice & ICU \\
2005 & 12 & 105.7 & 128 & 70 & Ice & Admit \\
2012 & 13.1 & 105.4 & 168 & 83 & IVF, Ice & Admit \\
2005 & 12 & 104.9 & 124 & 135 & Ice & Admit \\
2005 & 12 & 103.7 & 140 & 126 & Ice & Home \\
2005 & 12 & 103.7 & 108 & 114 & Ice & Home \\
2007 & 12 & 103.4 & 163 & 113 & Fans & Home \\
2008 & 12 & 102.8 & 140 & 110 & IVF, Ice & Home \\
\hline
\end{tabular}

aPresenting temperature was not listed on ambulance run sheet or in the initial ED record but was included in hospital discharge note.

CWI = Cold Water Immersion; Ice = Ice packs to groin, axilla, neck; IVF = Cool IV fluids;

Towels = Cold Towels; unk = unknown, MCD = medical cooling device 


\section{References}

1. Armstrong LE, Casa DJ, Millard-Stafford M, Moran DS, Pyne SW, Roberts WO. American College of Sports Medicine position stand. Exertional heat illness during training and competition. Med Sci Sports Exerc. 2007;39(3):556-572.

2. Armstrong LE, Crago AE, Adams R, Roberts WO, Maresh CM. Whole-body cooling of hyperthermic runners: comparison of two field therapies. Am J Emerg Med. 1996;14(4):355-358.

3. Bergeron MF, Devore C, Rice SG. Policy statement-Climatic heat stress and exercising children and adolescents. Pediatrics. 2011;128(3):e741-747.

4. Casa DJ, Armstrong LE, Ganio MS, Yeargin SW. Exertional heat stroke in competitive athletes. Curr Sports Med Rep. 2005;4:309-317.

5. Casa DJ, Guskiewicz KM, Anderson SA, et al. National athletic trainers' association position statement: preventing sudden death in sports. J Athl Train. 2012;47(1):96118.

6. Casa DJ, Kenny GP, Taylor NA. Immersion treatment for exertional hyperthermia: cold or temperate water? Med Sci Sports Exerc. 2010;42(7):1246-1252.

7. Casa DJ, McDermott BP, Lee EC, Yeargin SW, Armstrong LE, Maresh CM. Cold water immersion: the gold standard for exertional heatstroke treatment. Exerc Sport Sci Rev. 2007;35(3):141-149.

8. Cleary M. Predisposing risk factors on susceptibility to exertional heat illness: clinical decision-making considerations. J Sport Rehabil. 2007;16(3):204-214.

9. DeMartini JK, Casa DJ, Belval LN, et al. Environmental conditions and the occurrence of exertional heat illnesses and exertional heat stroke at the falmouth road race. $J$ Athl Train. 2014;49(4):478-485.

10. DeMartini JK, Casa DJ, Stearns R, et al. Effectiveness of CWI in the Treatment of EHS at the Falmouth Road Race. Med Sci Sports Exerc. 2014.

11. Harris PA, Taylor R, Thielke R, Payne J, Gonzalez N, Conde JG. Research electronic data capture (REDCap)--a metadata-driven methodology and workflow process for providing translational research informatics support. J Biomed Inform. 2009;42(2):377-381.

12. Hee-Nee P, Rupeng M, Lee VJ, Chua WC, Seet B. Treatment of exertional heat injuries with portable body cooling unit in a mass endurance event. Am J Emerg Med. 2010;28(2):246-248.

13. Kielblock AJ, Van Rensburg JP, Franz RM. Body cooling as a method for reducing hyperthermia. An evaluation of techniques. S Afr Med J. 1986;69(6):378-380.

14. Markenson D, Ferguson JD, Chameides L, et al. Part 17: first aid: 2010 American Heart Association and American Red Cross Guidelines for First Aid. Circulation. 2010;122(18 Suppl 3):S934-946.

15. Maron BJ, Doerer JJ, Haas TS, Tierney DM, Mueller FO. Sudden deaths in young competitive athletes: analysis of 1866 deaths in the United States, 1980-2006. Circulation. 2009;119:1085-1092.

16. McDermott BP, Casa DJ, O'Connor FG, et al. Cold-water dousing with ice massage to treat exertional heat stroke: a case series. Aviat Space Environ Med. 2009;80(8):720722. 
17. Muldoon S, Bunger R, Deuster P, Sambuughin N. Identification of risk factors for exertional heat illness: a brief commentary on genetic testing. J Sport Rehabil. 2007;16(3):222-226.

18. Proulx CI, Ducharme MB, Kenny GP. Effect of water temperature on cooling efficiency during hyperthermia in humans. J Appl Physiol (1985). 2003;94(4):13171323.

19. Roberts WO. Exertional heat stroke during a cool weather marathon: a case study. Med Sci Sports Exerc. 2006;38(7):1197-1203.

20. USA R. 2014 State of the Sport-Part III: U. S. race trends. Running USA. Accessed September 2014, 2014.

21. Wendt D, van Loon LJ, Lichtenbelt WD. Thermoregulation during exercise in the heat: strategies for maintaining health and performance. Sports Med. 2007;37(8):669-682.

22. Yankelson L, Sadeh B, Gershovitz L, et al. Life-threatening events during endurance sports: is heat stroke more prevalent than arrhythmic death? J Am Coll Cardiol. 2014;64:463-469. 